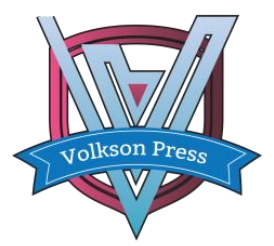

Contents List available at VOLKSON PRESS

Mechanical and Control Engineering (MCE)

DOI : http://doi.org/10.26480/wsmce.01.2017.99.101

\title{
DESIGN OF AUTOMATIC MECHANICAL AND ELECTRICAL EQUIPMENT MOTION PLAN FOR KELP NATURAL DRYING
}

\author{
Guangying Ma*, Chenyao Zhao, Daiyin Yang \\ School of Mechanical,Electrical and Information Engineering, Shandong University, Wenhua Xilu 180, Weihai, China. \\ *Corresponding Author Email: gyma@sdu.edu.cn \\ This is an open access article distributed under the Creative Commons Attribution License, which permits unrestricted use, distribution, and reproduction in \\ any medium, provided the original work is properly cited
}

\section{ARTICLE DETAILS}

\section{Article History:}

Received 02 october 2017

Accepted 06 october 2017

Available online 11 november 2017

Keywords

Kelp drying mechanism, bundling and cutting mechanism, automation equipment

\section{ABSTRACT}

In this paper, the automation electrical equipment of natural kelp drying is designed in the case of drying kelp difficultly. This equipment consists of four parts including rising transportation mechanism, drying mechanism, bundling and cutting mechanism and packaging mechanism. Because there has been a very sophisticated packaging mechanism on the market - the automatic sealing device, so the packaging mechanism will not be illustrated specifically. This paper mainly introduces the design concept of the first three mechanisms, specifically expounds their structure, composition, working principle, and the coordination of each other, at the same time, using AutoCAD and SOLIDWORKS software for two-dimensional design and three-dimensional modeling. At last, the application process and characteristics of the equipment are introduced for different conditions. This equipment is used to achieving automatic process of drying and harvesting kelps, which belongs to the integral assembly line production, can reduce labor intensity of workers and improve production efficiency of business.

\section{Introduction}

At present, the output of kelp aquaculture in our country ranks first in the world, and the developing momentum is swift. Nevertheless, the kelp industry in our country also has many issues, such as low resource utilization, low value-added of products, high production costs, poor economic efficiency, and serious environmental pollution and so on [1]

Especially in the kelp harvesting season, as the weather has more rain, kelp drying becomes a problem, many fresh kelps decay due to no timely drying and a lot of losses are caused to the enterprise [2]. But at present there is no complete automatic drying equipment of kelp at home, the latest drying frame of kelp is also artificial drying and does not include the binding and harvesting process. It is necessary to develop automatic mechanical and electrical equipment for easy drying, high reliability and high efficiency of kelp drying.

This paper presents a kind of kelp natural drying of automation electrical equipment which regards the automatic drying and bundling mechanism of kelp as the research object. This research work mainly includes the design of rising transportation mechanism, wheel support frame, transportation of wire in bundling and the whole device motion coordination and location etc. The working process of bundling and cutting mechanism mainly includes wire conveying, wire twisting, kelp cutting, wire cutting and device resetting, which is also the focus of the whole design.

\section{OVERALL DESIGN OF THE DRYING EQUIPMENT}

As shown in Figure1, the kelp natural drying of automation electrical equipment includes the following three main parts.

Drying mechanism: The effect is to hang the kelp on the drying rack, using the motor to drive the sprocket and chain, coordinating the track to make the kelp move slowly, achieving the natural drying effect.

Rising transportation mechanism: It is used to transport the kelp from the ground to the drying rack for the artificial hanging of kelp.

Bundling and cutting mechanism: Bundling and harvesting the dried kelp to achieve complete automation of the bundling and harvesting process, saving time and labor. The design of the internal parts is easy to disassemble and replace to improve the practicality of the equipment

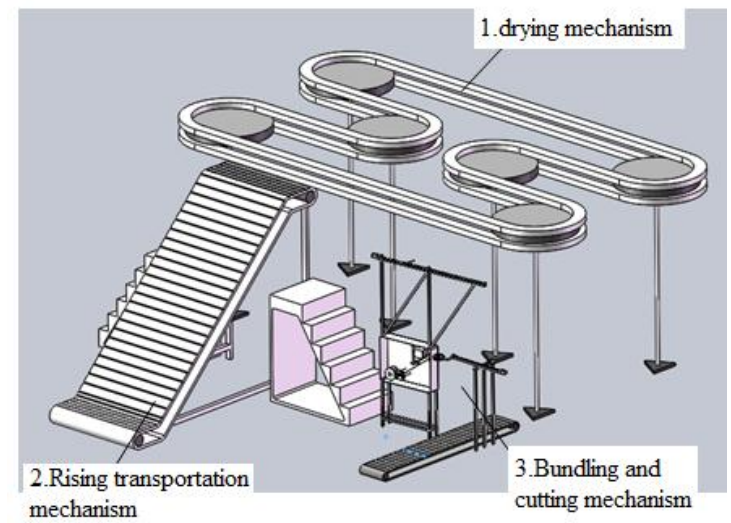

Figure 1: Composition Mechanism of Kelp Drying Equipment

The kelp drying equipment is mainly used during the day, covering a wide area and the use of time concentrating. Considering these practical factors, each part is designed to facilitate disassembly and assembly for transportation and operation in practical use, and the feasibility is higher.

\section{DESIGN OF DRYING TRANSPORTATION MECHANISM}

\subsection{Design Scheme of Drying Mechanism One (Double Tract Drying)}

Double track drying is to design the orbit into two inner and outer circles, kelp hung between the inner ring and outer ring and driven by the sprocket and chain. This track is like a return ring of similar "gong" word [3]. The orbit of this shape is adequate for the use of space. In order to facilitate roller rolling, the track is designed into a concave groove.

Because the kelp needs to be hung, and the belt is not enough to carry weight, conveying not operating at a low speed, circulating circularly the goods in the horizontal plane, this design chooses the chain to drive. Chain conveyor can realize the above functions, and the structure of the chain can be changed, as well as it has the advantages of stable running speed and low power consumption during the working process $[4,5]$. 
The conveying of drying mechanism is designed as single transportation chain for the double track conveying. In the design of the chain, the chain connecting shaft is extended installing a roller shaft on the connecting shaft, and respectively installing a roller on the two ends of the shaft. This roller shaft is used to hang the kelp, as shown in Figure 2(a). (Assuming the kelp evenly distributes the load, the gravity of the kelp is distributed evenly to the roller on both sides of the axle).

The sprocket which is matched with the chain has no excessive precision requirement, and only needs to drive the chain. The whole assembly diagram of drying mechanism as shown in Figure 2(b).

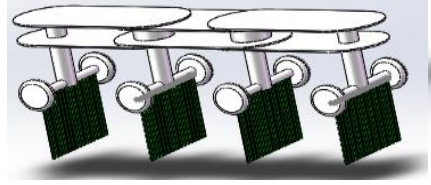

(a) Suspension of Kelp on Roller Axle

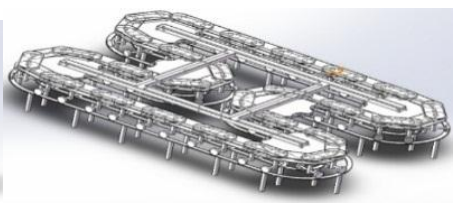

(b) the Whole Assembly Drawing
Figure 2: Drying Mechanism of Double Tract Drying

\subsection{Design Scheme of Drying Mechanism Two (Single Track Drying)}

There is only one orbit for the single track drying, and the kelp seedling rope is parallel to the track. To facilitate roller rolling, both sides of the track are designed into concave grooves. The chain schematic diagram is shown in Figure 3(a). The middle of the sprocket passes through a square axis, and at the bottom of the shaft is a ring that is used to hang the side of the kelps. The upper end of the shaft is equipped with two rollers which are rolled in the upper orbit.

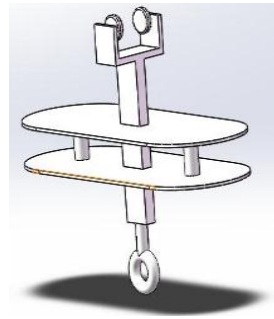

(a) Single Chain Design

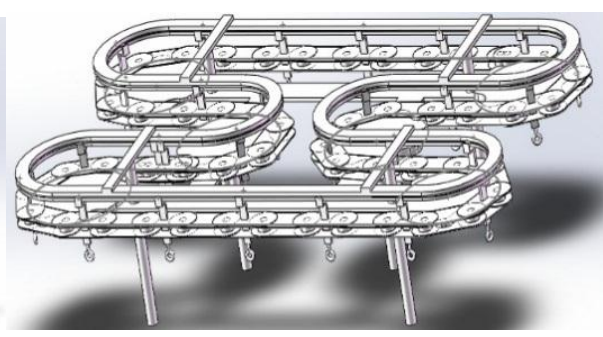

(b) the Whole Assembly Drawing
Figure 3: Drying Mechanism of Single Tract Drying

Sprockets and chains are in meshing, and pitch selection is also determined by non-standard chain. Sprockets are arranged in the center of the curve of track, selecting one of the sprockets to install the motor, to become the driving sprocket, driving all the sprockets, thus driving the circular drying of the kelp. Its overall assembly diagram is shown in Figure 3(b).

\subsection{Scheme Selection}

The advantages of scheme one is that the number of drying kelp is more and the area is smaller. Its disadvantages are that the light in the inner circle is always less, and it is not guaranteed to be evenly dried, which may affect the quality of kelp. The advantages of scheme two are more uniform drying, but the disadvantages are larger floor area. When the number of kelp is larger, choose scheme one, when the number of kelp is smaller, choose scheme two.

\subsection{Design of Support frame}

Due to kelp drying needs the large space and place, it is generally chosen on the smooth beaches or unattended roads. It is necessary to design different support frames to meet the requirements of the actual site.

The triangular plane is used to increase the force area and prevent the support frame from being overloaded into the sand. The three fixed posts under the plane are used to insert the position of the fixed equipment in the sand, so that the support frame does not move and improve the stability, as shown in Figure 4. This equipment that placed on the road do not consider the problem that the support frame sinks, so directly using the stable tripod support structure can make the equipment stable, as shown in Figure 5.

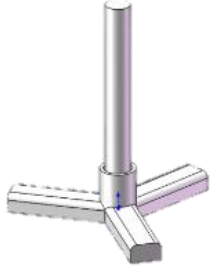

Figure 4: Beach Support Frame

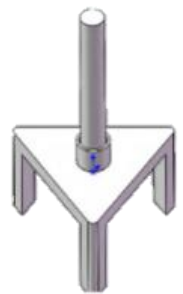

Figure 5: Highway Support Frame

\section{DESIGN OF RISING TRANSPORTATION MECHANISM}

\subsection{Composition of Rising Transportation Mechanism}

The purpose of rising transportation mechanism is to transport the kelp with a seedling rope from the ground to the hanging ring of the drying mechanism to facilitate hoisting the seedling rope to the drying mechanism artificially. The driving device uses the belt elevator (belt drive), using the motor to drive the sprocket and the chain, coordinating the corresponding support frame [6]. Since the suspension process requires manual operation, it is necessary to cooperate with the corresponding workbench on both sides of the belt hoist to facilitate the operation of the workers. The overall assembly diagram is as the lower left corner shown in Figure 1.

\subsection{Working Process of Rising Transportation Mechanism}

The kelp with seedling rope is placed on the platform below the belt elevator and two ends of the seedling rope are entwined round the protruding places on both sides of the conveyor belt (not shown in the picture) to prevent the fall of the kelp. The conveyor belt is sent to the upper platform by the motor, and then the seedling rope is disengaged by the workers standing on the worktable to hang on the hanging ring of the drying mechanism manually. When harvesting the kelp, the harvested seedling rope is also artificially disengaged and placed on the conveyor belt downward transportation.

\section{DESIGN AND OVERALL ASSEMBLY OF BUNDLING AND CUTTING MECHANISM}

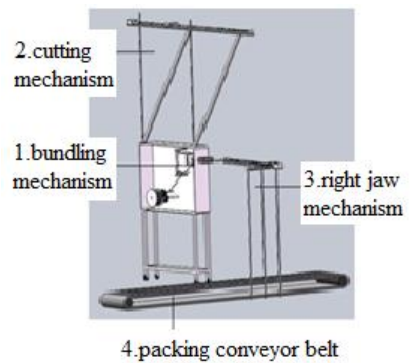

(a) the Overall Structure

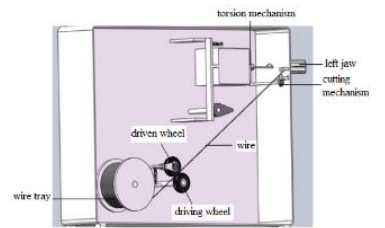

(b) the Bundling Mechanism

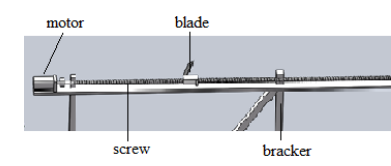

(c) the Cutting Mechanism

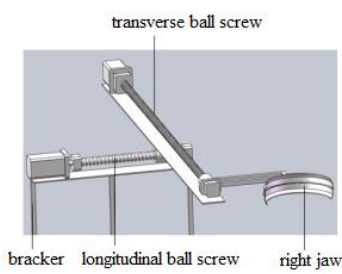

(d) the Right Jaw Mechanism
Figure 6: Composition of Bundling and Cutting Mechanism 


\subsection{The Bundling Mechanism}

As shown in Figure 6(a), the bundling and cutting mechanism consists of four parts including bundling mechanism, cutting mechanism, right jaw mechanism and packing conveyor belt. The bundling mechanism (as shown in Figure 6(b)) mainly completes the action that wire wraps kelp, whose purpose is to realize the complete automation which the wire wraps kelp. Bundling mechanism includes the wire coiling plate for receiving the wire, the sleeve for controlling the wire guiding, the jaw (channel wire slot on the inside) for bundling kelp, the main driving wheel for driving the wire and the coordinating rubber wheel for pressing wire, the torsional framework for reversing the wire to tighten the kelp and cutting mechanism for cutting wire after the completion of bundling.

\subsection{The Cutting Mechanism}

The cutting mechanism (Figure 6(c)) mainly completes the cutting of the kelp and is a whole with bundling mechanism. After the kelp is bundled tightly, the cutting mechanism completes the cut of the kelp. This mechanism requires long stroke and fast speed, using the low power servo motor to drive the horizontal ball screw for the fro movement.

\subsection{Right Jaw Mechanism}

The above design is the left half part, and the corresponding right half jaw and driving device should be designed, as shown in Figure 6(d). The right jaw and the left jaw can be integrated into a full circle, opening wire slot in the jaw to ensure that the wire can bundle tightly the kelp inside the jaw. The right jaw requires transverse and longitudinal motions, using the servo motor to drive the corresponding ball screw to complete the forward and backward actions of the jaw.

\subsection{Packing Conveyor belt}

A 1.8-meter-long carton is placed on the conveyor belt. When the kelp ......, the conveyor belt moves rapidly forward or backward, and the kelp is folded in half and fell into the carton by gravity and inertia. After reaching a certain weight, the conveyor belt will convey forward, and the carton is sent to the packing mechanism.

\section{WORKING PROCESS OF THE KELP DRYING EQUIPMENT}

The drying of kelp is divided into three working hours in one day, and the working tasks in the early, middle and evening are different, using different combination of the mechanisms.

In the morning, the kelp starts to be dried. The kelp is transported by rising transportation mechanism from the ground to the drying rack, the workers that stand in the workbench hanging the seedling rope to the hanging ring of the drying rack manually, drying rack rotating a certain distance for the hanging of the next seedling rope. After using the equipment, the rising transportation mechanism will be removed. Only the drying mechanism will be operated during the day, the operating speed will be controlled to meet the natural drying of the kelp. In the evening, the kelp is harvested. The kelp rotates and moves to the bundling and harvesting mechanism, bundling mechanism wrapping the kelp with the wire, the torsional framework tightening the wire, resetting the right jaw after harvesting mechanism cutting the kelp, falling on the conveyor belt. When the kelp is harvested, the seedling rope is moved to the rising transportation mechanism, which is disengaged by works and sent to the ground.

\section{CONCLUSION}

This new kind of kelp natural drying equipment can meet the needs of the work well and each part is easy to disassemble and operate independently, completing automatically the drying, bundling and harvesting of the kelp. This paper designs and summarizes the kelp natural drying equipment, researching and analyzing bundling and cutting mechanism and drying mechanism of the kelp natural drying equipment, expounding the respective working principle and working process, arranging two design schemes and making comparisons and choices.

There is also optimization space for the kelp drying equipment in the structure, such as further shortening the bundling and cutting time of the single orbit, improving the stability and reliability of the kelp drying equipment.

\section{ACKNOWLEDGMENTS}

We gratefully acknowledge the financial support provided by the special fund of the Institute of marine research of University Co-Construction in Weihai city.

nemonerimon

1 . . belt conveyor. Coal Mine Modernization, 1009-079701-0074-02, 74.

[2] Zhenhui, J. 2009. The status and development trend of China's kelps. Transactions of Oceanology and Limnology, 01-0141-10, 142-149.

[3] Li, J. 2007. Design of automatic chain conveying equipment. Industrial Technology, 830001,1.

[4] Lianggui, P., Minggang, J. 2006. Mechanical design. The eighth edition, Beijing, Higher Education Press, 165-185.

[5] Qina, W. 2010. The problem of kelp drying. Meizhou Daily, 06-03,1. Shensheng, Z. 2004. The principle and application of linear guide. Hongdu technology, 2, 45-49. 